\title{
SIMULATION-BASED PERFORMANCE ANALYSIS FOR FUTURE ROBUST MODULAR PRODUCT ARCHITECTURES
}

\author{
Dambietz, Florian M.; \\ Greve, Erik; \\ Krause, Dieter \\ Hamburg University of Technology
}

\begin{abstract}
The increased demand for customer-adapted product solutions shows an increasing trend of product variety, leading to an increased internal variety and therefore -costs. The concept of modularization provides apossible solution to this challenge by developing modular kits. Nevertheless, modularization methods to not lead to one individual modular kit, but to several alternatives. The decision of which alternative to implement can be crucial to the applying companys succes. During this decision-making both customer- and company perspectives need to be taken into account. This contribution is to present a simulation-based approach to support the decision making by using a model-based configuration system. Furthermore, as classical decision-making processes are based upon historical data, future aspects are usually not taken into account. In order to counteract this situation, this contribution intends to simulate as well future aspects impacting the modular product architecture. In this case, the simulation is used in order to evaluate the individual performances of a Design-forVariety product architecture as opposed to a Design-for-Future-Robustness by applying this method to the example of customer-individual laser machines.
\end{abstract}

Keywords: Simulation, Design for Future-Robustness, Product architecture, Configuration, Constraint modelling

\section{Contact:}

Seiler, Florian Michael

Hamburg University of Technology

PKT

Germany

florian.seiler@tuhh.de 


\section{INTRODUCTION}

In the course of globalisation, manufacturing companies are confronted with a continuously increasing competitive pressure. As a result, many market players feel compelled to intensify specialisation in order to be able to respond to customer-specific requirements. At the same time, a high degree of standardisation is required to meet the high-cost competition. In this case, internal variety management is a core task to ensure competitiveness. One possible solution to meet this challenge is provided by modularity. Here, the focus is on reducing internal variety while keeping external variety towards the customer as high as possible. Within the framework of modularisation, company-specific product families with the corresponding set of rules are developed in order to be able to react flexibly and dynamically to customer enquiries.

The design of modular product families is a decision that has a long-term impact on the entire company (Krause \& Gebhardt, 2018). Besides the operational character of the actual product family design, essential strategic goals are also linked to this product structuring strategy. For example, a modularized product family can help to bind new suppliers in the long run or to achieve a more flexible market position and thus to an increase in competitiveness. In order to be able to exploit these long-term effects, it is often necessary to make extensive process adjustments and organizational changes in the company. Their implementation depends on the resources used and often takes a long time. During this time, changes can occur which lead to cost-intensive adjustments to the initially developed modular product architecture (MPA) and result in the fact that the intended potentials cannot be exploited. These changes must be anticipated in the early planning phase and taken into account in the MPA design. Such modular product families are designated as future robust (Greve \& Krause, 2018).

When developing these individual product architecture alternatives, one of the main difficulties is the requirement of focusing the development process on various target values simultaneously. This task is often not methodically supported, leading to the decision as which of the product family alternatives is finally to be implemented being made on the basis of a few influencing factors. Additionally, the alternative selection is often based to a large extent, on expert decisions. Nevertheless, this decision is crucial to the modular kit's and therefore, the company's success.

With the combined field of customer-, market-, and company perspectives forming a strongly dependant field of analysis dimensions (Cicconi et al., 2018), a methodical approach to this conjoint target value-oriented decision-making process is required. At this stage, the underlying contribution proposes a solution to filling this gap. In order to provide a quantifiable and holistic criterion to support the selection of modular kits, a multi-factorial simulation is used. One of the decisive aspects of this is the mutual consideration of both customer and company perspectives. A decision support method based upon retrospective historic customer data is presented by the configuration-based simulation performance analysis as described in Seiler et al. (2020b). By integrating cost-effective and maintainable aspects as well from the customer-, market-, and company perspective, a quantitative decision-support index can be generated during the early development stages.

The major deficiency with this stated method is its assumption, that customer requirements and therefore the corresponding product architectures stay the same when considering future business evolvement. At this point our contribution aims at filling that gap by integrating a method for futurerobust design into the simulation data basis generation, being based on the hypothesis of improving reliability and applicability of the simulation's output. Future changes to the product architecture can thus be reduced and long-term potential can be exploited. In order to assess the degree of importance considering future-robust design, a compared analysis of future robust product architectures towards common variant-adequate product architectures in relation to the existing product architecture's status quo can be carried out.

Our contribution is three folded. The first part is to explain the simulation-based performance analysis as a method of assessing future robust product architecture alternatives. By using a simulation-based method, multiple scenarios as well as product architecture alternatives can be evaluated in a costeffective way, allowing for an early-stage decision support. Secondly, the conjoint impact of multiple relevant analysis dimensions, as in this case the Degree of Variance, Need for Change, Change Effort as well as e.g. Manufacturing Cost can be analysed. And thirdly, the trade-off between usually more complex future-robust and the common variant-adequate product architectures impedes large difficulties when intending to validate the advantage of future-robust product architectures. The presented simulation provides a possible solution to mathematically and graphically display the 
differences in their individual performances. In detail, the combined assessment of customer- and company perspectives is made possible by using a configuration system as a core simulation tool. For explanation purposes, the method is displayed using an individual module from a modular kit for laser processing machines.

\section{STATE OF THE ART}

In the following, the topic of future robustness is addressed and the method used to design the product architecture concept alternatives to be evaluated is briefly presented. Furthermore, a brief description of the used simulation-based performance analysis is given.

\subsection{Future robust modular product family design}

For the design of modular product families to handle the diversity of product variants, there is a multitude of tools and methods available in the literature (Krause \& Gebhardt, 2018). The aim of the methods is to affect the modularity of a product architecture in such a way that functional and/or product-strategic advantages can be achieved. Modularity can be considered as a gradual characteristic, which, according to Salvador (2007), can be described by the attributes of decoupling, communality, combinability, functional binding and interface standardization. Hackl et al. (2020) add the attribute of oversizing to this characteristic. Especially communality and combinability are the relevant levers for cost savings across product variants (Hackl et al., 2020).

The orientation of a modular product family must always be based on the customer-relevant product features, as these are the ones that bring the company the benefit (Kipp \& Krause, 2008). The customer consciously perceives these product characteristics and incorporates them into his/her decision-making process for selecting a product variant. The principle of Design for Variety (DfV) addresses this circumstance and has the goal of obtaining a minimum internal variety at components and processes by the configuration of a fixed external variety (Kipp \& Krause, 2008).

Due to the dynamics mentioned at the beginning, however, it is no longer sufficient to handle only the present product variety. In fact, the diversity induced by future changes must also be taken into account in order to be able to exploit the advantages of a modular product family in the long term (Greve and Krause, 2018). This circumstance can be addressed by the changeability of a product architecture. The objective of this approach is to be able to react to unforeseen changes initiated by dynamic influencing factors. For the design of MPAs in efficient handling of changes, the concepts of robustness and flexibility are particularly effective (Bauer, 2016). Such a design concept enables the elements to be reused across product variants and to achieve long-term economies of scale. In addition, not only the risk of a dynamically caused change is minimized, but also the acceptance of the risk is allowed by reducing or eliminating the negative change effects (Bauer, 2016). Despite changeinducing uncertainty due to dynamic influencing factors, subsequent changes can be made with reduced costs and effort.

The consideration of the expected variety induced by future changes can be designated as Design for future Robustness (DfFR) (Greve \& Krause, 2018). The main drivers for future changes are customer requirements and production-specific factors, such as change of production processes or suppliers. They lead to cost-intensive adjustments to the MPA and to the fact that the long-term potentials (which also occur in production) cannot be fully exploited. In the literature, there are very few approaches that simultaneously consider changes in customer and production requirements in the design of MPAs. The focus is mostly just on customersand is limited to a conceptual design of the module limitations (comprehensive overview of methods and analysis with the present research focus can be found in Greve \& Krause (2018)).

In this paper, the approach of Greve et al. (2021) is used, with which the modular product family can be redesigned on the component level so that it is robust against future changes in customer and production requirements. The central element of this approach is the Change Allocation Model (CAM) - a visualization that enables the identification and redesign of the change-critical elements of the product family on the basis of decision-relevant criteria (change effort, change requirement, existing variance). The results of the method are alternative product architecture concepts, which are characterized by the fact that they are as robust as possible against future changes. 


\subsection{Product architecture performance analysis}

The main task of the proposed method is to identify the most suitable and efficient MPA of all identified alternatives within the modularisation process. Moreover, since each company operates in a different business environment and for different customers, the influencing factors must be taken into account. These factors create the relevant and appropriate simulation framework.

The first step is to transform the unstructured product architecture into a MPA by using modularization methods in the initial modularization phase. As already described, different methods lead to different MPA alternatives. On this general level, it is not important to define an individual modularization method. For the current explanation of this method, the Integrated PKT-Approach for the Development of Modular Product Families according to Krause \& Gebhardt (2018) is used as a combination of both functional and strategic design methods.

The resulting MPA 1...n from the initial phase of modularization determines the total sum of alternatives, among which the most efficient should be determined. So far, the most modern process has come down to experts who use classified knowledge and a limited number of analytical factors, such as, for example, the number of standard components within a given alternative, in order to obtain a qualitative result for the best performing alternative.

The following figure 1 displays the generic set-up of the proposed simulation performance analysis method, integrating a product configuration system as a core element according to (Seiler et al. 2020b).

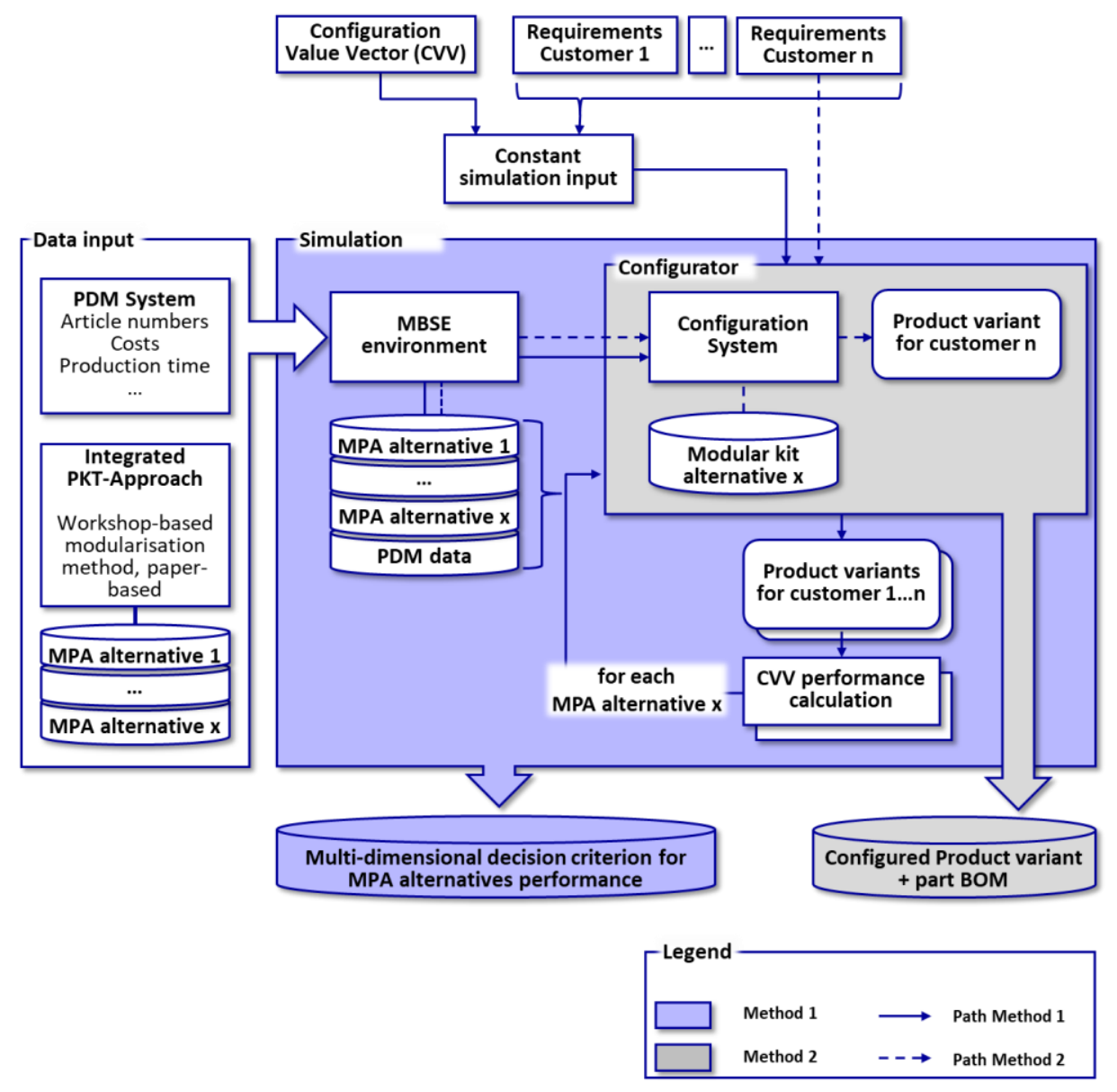

Figure 1: Generic simulation set-up on the basis of Seiler et al. 2020b

As these MPA alternatives consist of a huge amount of data, with a large number of dependencies and constraints to be taken into account, depending on the complexity of the product, and the business and customer environment of the company is described in many different variables, the only sensible way to process these large amounts of data seems to be by applying a computer simulation. Therefore, once all the different variants have been identified, simulating these variants while taking into account both customer and business environment factors is the next step to form the basis for the next phase of the multi-factor assessment. This leads to a final decision on which alternative should be implemented 
This is mainly made possible by the use of a dynamic product configuration system. As an interface for translating the customer perspective into the company perspective, it enables an intuitive transfer of the knowledge explicated by the customer within the framework of modularisation. By prioritising different influencing factors such as costs, delivery time or the expected design effort, the customer and the company can influence the final determination of the configured product variant (Zennaro et al., 2019). These configured module variants possess each a combined set of performance values, described as Configuration Value Vectors (CVV, see Seiler et. al, (2020b)). These values represent the individual module variants' performances and can then be linked using a multi-dimensional hyperspace calculation approach according to (Seiler \& Krause, 2020a), which basically considers the individual configuration paths as a geometric optimisation problem with the analysis dimensions providing the framework for the geometric space. By calculating the spatial area of these geometric configuration representations, a performance measurement index can be derived.. In order to keep the underlying, partly complex product architectures consistent and maintainable, the use of a model-based data structure is additionally demonstrated. The use of the Model-Based Systems Engineering (MBSE) approach helps to express the complex interrelationships of the modular system in a consistent and machine-readable form (Stjepandic et al., 2018). Thus, the configuration system can access the ontology of the underlying data structure independently from the product. For the modular system simulation, this configuration system is recursively used for several customer requests and alternative modular systems, in order to subsequently generate a multi-dimensional decision criterion regarding the modular system performance by means of a geometric-mathematical algorithm (Seiler et al., 2020b).

\section{USE CASE DESCRIPTION}

The during this explanation used product example consists of one individual module from a modular kit for laser processing machines. This module describes an indexing table, which is generally used for maintime-parallel loading, enabling on the one side the loading of pre-parts, while on the other side the parts are processed. When the process is finished on the inside of the machine, the indexing table rotates by $180^{\circ}$ and therefore exchanges the processed part with a loaded pre-part. Furthermore, in order to shield the operator from potential hazardous laser radiation, both indexing table sides are separated by a separating wall enclosed by two beam traps and a horizontal hatch. The following figure 2 displays a graphical representation of the indexing table's status quo before the modularization process.

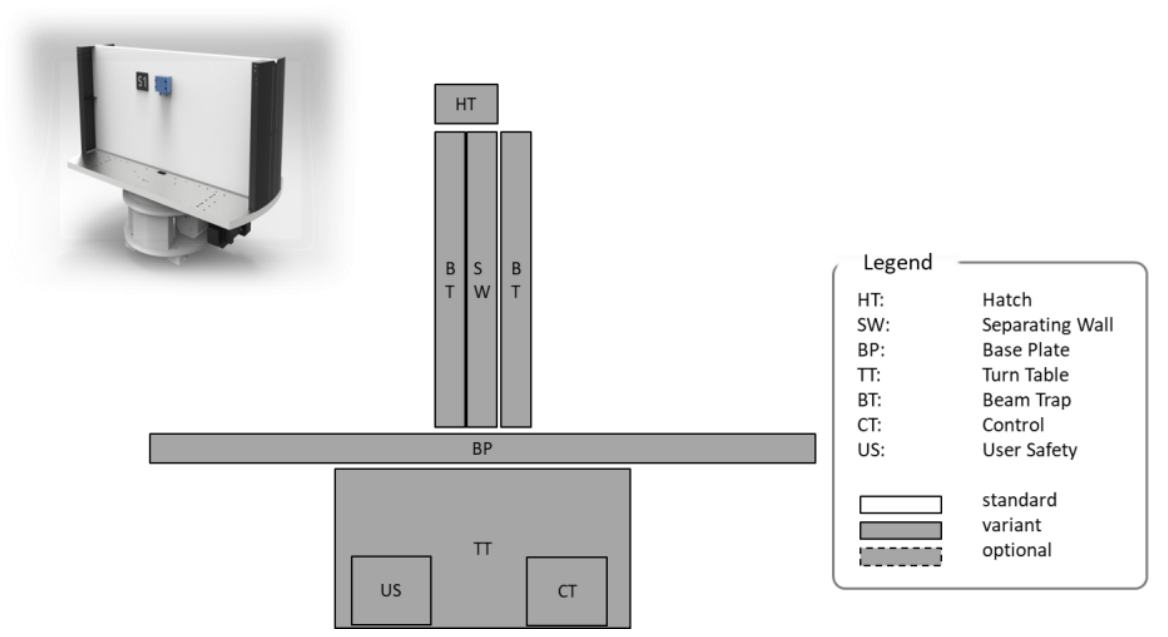

Figure 2: Turntable product example: real product (left upper corner) and Module Interface Graph (MIG) of status-quo product architecture

The variety of the module is displayed using the Module Interface Graph (MIG) according to Krause \& Gebhardt (2018). The individual components the module is composed of are schematically displayed in order to give the use a brief overview to their geometric shape and placement. Furthermore, their degree of variance is expressed by the colour of the individual component. Standardized components are visualized in white, whereas variant components are stylized in grey. Therefore, the status quo as displayed in figure 2 contains only variant components. This is due to the reason that the main defining part, the base plate is functionally linked to all other components as they 
are either diameter- or weight dependant. These two issues are both strongly linked to the base plate. This results in the components separating wall, beam trap, hatch and turntable evolved as variant components, leading to variant software control and user safety modules. As this variance is commonly considered as majorly impacting a product architecture's performance, a modularization process has been conducted, leading to a DfV-alternative as displayed in the following section. Additionally, a future-robust indexing table alternative is displayed as an outset point for the performance comparison simulation.

\subsection{Definition of alternative modular product architectures}

In the following figure 3, the DfV alternative (left) as well as the DfFR alternative (right) are displayed. As it can be seen as opposed to figure 2, the DfV product variant (figure 3 left-hand side) has a significantly increased degree of standardization, majorly resulting from an over-dimensioning of the turntable and control components (symbolised in white). By meeting the same customerrelevant properties as the status-quo alternative, the external variety is kept at the same level, whereas the internal variety has already been reduced.

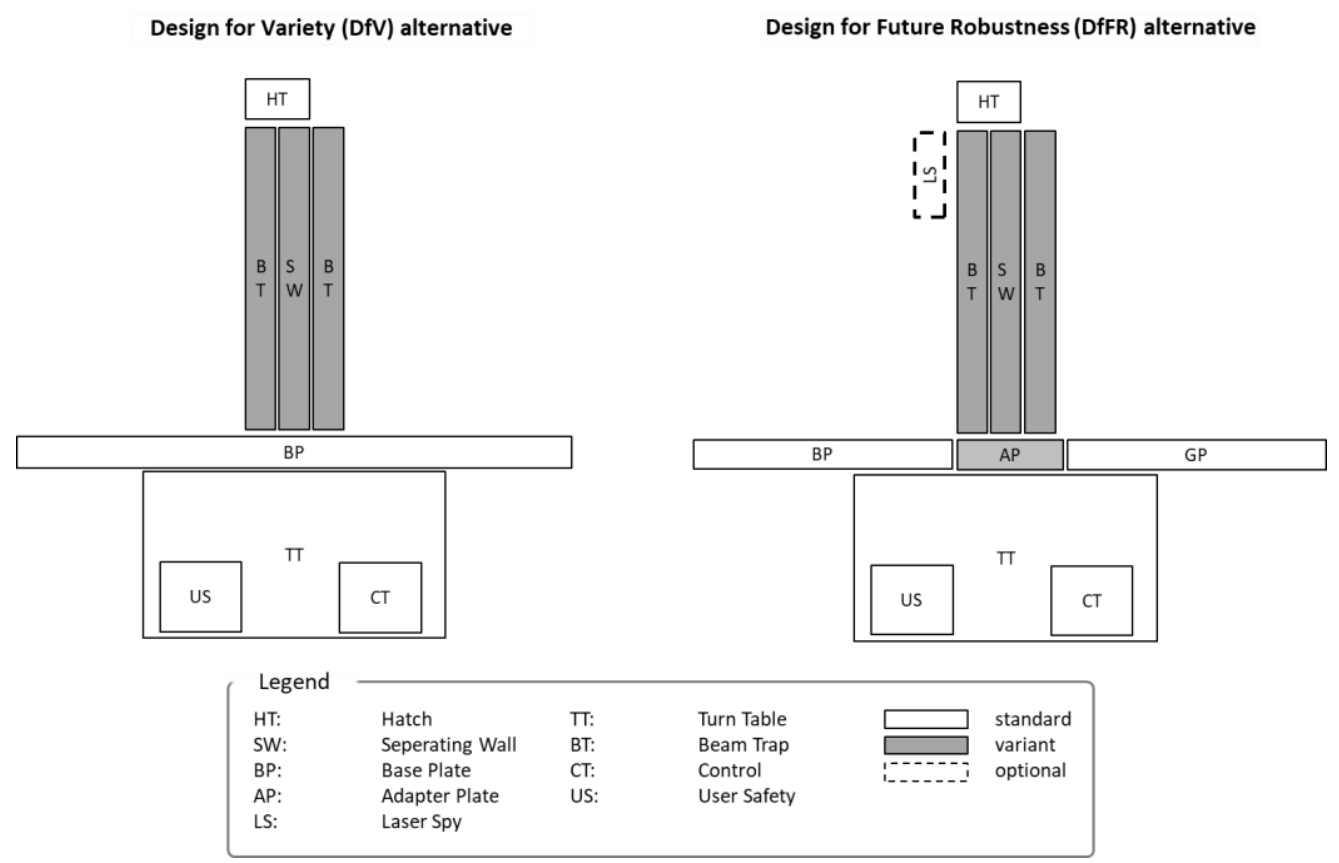

Figure 3: MIGs of DfV-alternative (left) and DfFR-alternative product architecture (right)

Nevertheless, an exemplary additional customer requirement and therefore customer relevant property has been identified for the future market and customer evolution. In this case, it is considered as highly likely, that customers demand for an Active laser safety system instead of the existing Passive laser radiation shielding. In order to meet this additional future customer-relevant property, the DfFR alternative (figure 3 right-hand side) possesses an additional standard, but optional component (marked as white and dash-lined). This component Laser Spy contains an optical sensor unit required for an Active laser safety system. As a result, the DfFR alternative is able to meet future customer requests to a higher extent than the DfV alternative. Furthermore, the base plate component is split into two separate components, introducing an Adapter plate in order to further reduce the variance impact. Additionally, as future scenarios in this example show a strong demand for increased rotation speeds, the turntable component in the DfFR-alternative is further over-dimensioned as compared to the DfV-alternative, leading to an increase in its manufacturing costs. Both these changes result in meeting more future customer request expressions, but come with the trade-off of increased manufacturing costs.

Additionally, the production processes for individual components varies between the above-stated alternatives. Especially for the base plate component, these changes on the production side are applied. In this case, the original casting process is replaced for the DfFR alternative by laser cutting the rough shape and drilling the remaining fixing threads. By applying these changes, a significant reduction in cycle times, flexibility and, most important, production depth can be achieved. This furthermore leads to a reduction in this component's production cost. 


\subsection{Determination of analysis dimensions}

As described during section 2.2, the simulation requires a parameter framework which defines the analysis dimensions under which the module alternatives' performances are to be assessed. In this case, five relevant factors have been identified and are described in the following.

- Configuration Depth

This particular factor describes the ratio of met or not met customer requests by the configured product variant. Therefore, the modular kit's capabilities define the maximum achievable configuration depth. If every single customer request can be met by a hypothetical product variant, the configuration depth is set at $100 \%$. For every not met customer request, the configuration depth decreases according to its weighted impact (Seiler \& Krause, 2020a).

- $\quad$ Variance

The degree of variance describes the ratio of variant and standard components with respect to the total amount of components within a MPA. In case of the standard components, a further differentiation is performed due to two separate time perspectives. "Regular" standard components are defined if they represent a standardised component according to the retrospect view and are usually the result of a variety-oriented product architecture development. When it comes to future-robust product architectures, these retrospect standard components are susceptible to changes and can therefore induce variety on a future perspective. Accordingly, future-robust standard components are not only standardized in a retrospect view, but also standardized according to future view. This type of components is considered das the most advantageable sector.

- $\quad$ Need for Change

The need for change can basically be seen as a probability factor, according to which changes to the individual component are likely to appear. If a high probability for future changes is identified according to the applied future scenario method, the need for change index is considered as significantly high.

- $\quad$ Change Effort

This factor quantifies the effort which needs to be taken in the future in order to counter the results of the expected changes in the customer relevant properties, the marked field or the individual company's perspective. The change effort is strongly linked to the amount of individual linkages originating from the considered component to other components. If this linkage number is high, applying changes to the considered components results in a required change of the linked components, leading to a significantly high change effort.

- $\quad$ (Manufacturing) Cost

In this case, the cost factor is mainly formed on the basis of the manufacturing costs for the individual components within the MPA. It is considered as relevant, as the process of standardisation comes with varying degrees of over-dimensioning. This is the case also within common variant-oriented but especially within future-robust product architectures and therefore represents the possible negative performance impact of future-robust product architectures.

These analysis dimensions are implemented into the simulation method as individual values of the above-described CVV, forming the geometric space of the configuration path representation. By attributing individual values to these framework parameters, a conjoint and detailed analysis on the MPA's component level can be carried out. Furthermore, a set of customer requests is needed as an additional simulation input. These customer requests, if not originating from historic data, can be derived using several future scenario techniques (Fink \& Siebe, 2016). In this case, a set of ten customer requests have been identified which then serve as an input for the simulations central core, the configuration system. At this point, it is of major importance to state that these customer request form the constant input for the upcoming performance analysis simulation. This means that all three module alternatives (status quo, variant adequate and future-robust) with their individual set of components, degrees of variety as well as differing meeting customer relevant properties are simulated with the same sets of customer requests. This leads to a performance index, which allows for a comparison of the individual module alternatives under the estimated future customer- and market premises. 


\section{SIMULATION AND PERFORMANCE ANALYSIS}

The simulation itself is implemented on the basis of an MBSE-data structure. This enables a consistent and change-robust data structure for the MPA, the containing components, customer-relevant properties, modules and module variants (Kovalenko et al., 2019). This is displayed following figure 4 with an excerpt of the modelled Configuration Network Diagram (CND).

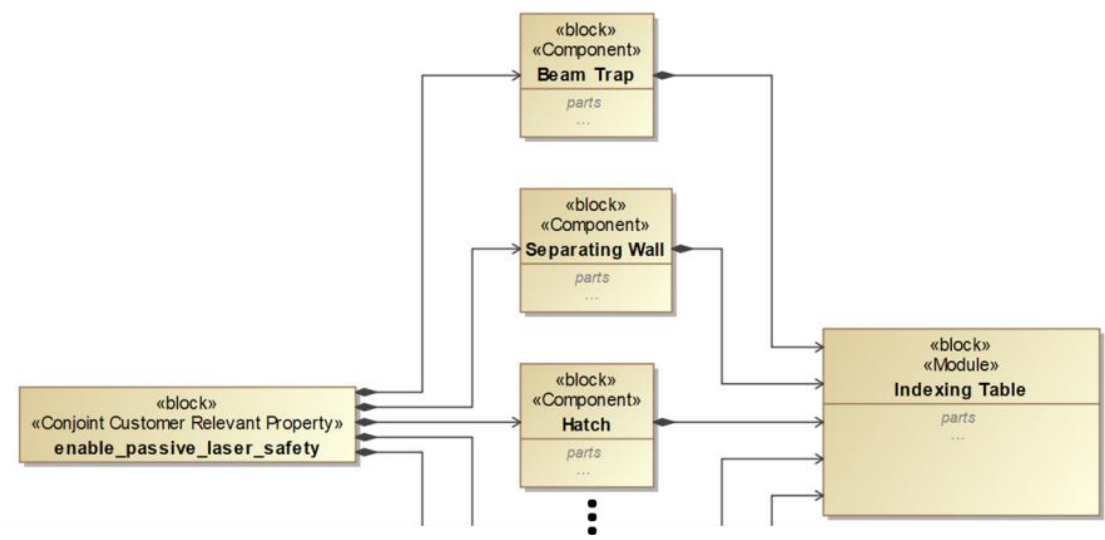

Figure 4: Excerpt of modelled MBSE-Configuration Network Diagram

The CND is used in order to link customer-relevant properties to components clustered into individual modules by allocating Block-Items of the corresponding stereotype as a Block-Definition-Diagram). This data structure is then used as the basic data core as well as constraint-defining baseline for the product configuration system (James et al. 2016), which forms the central tool of the applied simulation performance analysis tool. Furthermore, a configuration frontend has been designed in order to enable the translation of the customer perspective (expressed by customer requests) into the company perspective (expressed by the customer-relevant properties). The major important part of the simulation is represented by these input customer requests, as they form the consistent simulation baseline. In this case, a total amount of ten sets of customer requests have been identified by applying a scenario method. Therefore, the customer requests represent expect future customer requests, taking into account relevant changes in the market-, customer-, and company environment. Having identified these sets of customer requests, the simulation is carried out on the basis of the three described module alternatives as shown in the above sections. Hereby, at first the status-quo module alternative is used as a data core. On this basis, the ten sets of customer requests are used as a configuration input, leading to ten sets of more or less matching product variants. The same procedure is repeated analogically for the DfV and DfFR module alternatives, leading to in total three different sets of ten configured module variants each.
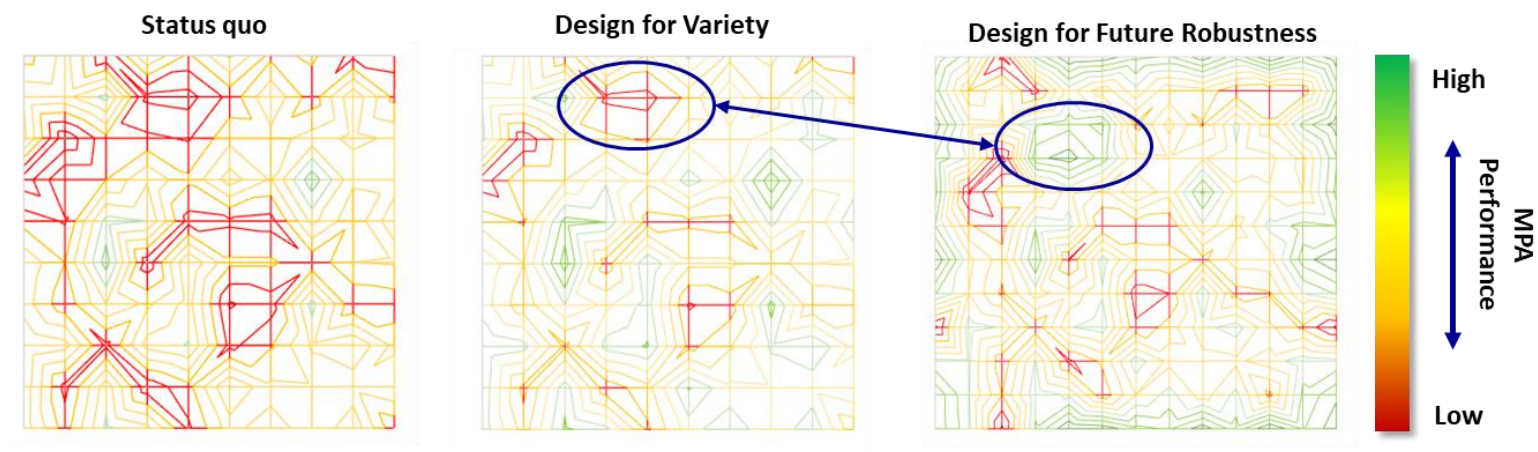

Figure 5: Heatmap simulation results for indexing table architecture alternatives with marked future customer request match/mismatch (encircled areas)

The exact derivation of the most performant alternative is done not by using the heatmap, but by referring to the actual integrated hyperspace values. These heatmaps are just an additional graphical representation of the multi-dimensional simulation result. By using an euclidian projection, 2D-displays are generated, leading to non-descript axes as they include multiple analysis dimensions each. As the heatmaps of the above figure 5 display, the heatmaps for the status quo module alternative and the DfV- 
alternative show similar geometric heatmap patterns, but with differing performance indices. In this case, the DfV-alternative shows a significant performance increase, as the large red areas are shifted towards yellow or green areas. This performance increase is due to the increased standardization level and therefore, the reduction of the internal variety. This already shows the positive effects of the DfV.

Another important fact to be stated considers the heatmaps' sizes. The left and middle heatmpas have the same size, as the amount of customer relevant properties as well as the number of used components stays the same when comparing the status quo and the DfV-alternatives. On the other hand, the DfFRalternative on the right-hand side of figure 5 shows a larger size. This is due to the fact, that an additional expected future customer relevant property and accordingly, additional components (the laser spy component) are integrated into the product architecture. Furthermore, as the MIGs above display, the base plate component is split into several individual components in the DfFR-alternative when compared to the DfV-alternative in order to reduce the impact of the occurring variety. When it comes to analyse the relevance of the additionally integrated future customer-relevant property, the encircled areas in figure 5 need to be regarded. The performance index for this to be met future customer-relevant property is stated as a low-level performance and therefore marked as red in the DfV-alternative. This is due to the not implemented laser spy component, which can meet the additional customer-relevant property. This changes when regarding the DfFR-alternative, where the encircled area shows the same impact as for the DFV-alternative at the same heatmap-location of this customer-relevant property. In the case of the DfFR-alternative, the performance level is considered as high and therefore marked in green, as the additional integrated component can meet the additional customer-relevant property.

Furthermore, the DfFR-alternative is a bit less performant in the centre of the heatmap, which is representing the same components as with the DfV heatmap. This is due to the over-dimensioning and the thereof resulting increase in manufacturing costs when intending to implement future-robust standard components. But all in all, when analysing the trade-off between met customer requests and manufacturing costs, a clear advantage of the DfFR alternative can be seen. Especially the overdimensioning of turntable has neglectable negative performance impact, as only the material costs increase slightly, which is in no relation to the change effort required when designing a hypothetical new future variant.

\section{DISCUSSION}

According to the above-shown simulation results, a clear difference from the status quo performance towards the DfV and the DfFR alternative architectures can be stated. The results generated by the simulation match the mental model of the represented use case, enabling a first validation of the overall results. Nevertheless, it needs to be stated that these results are strongly dependant from input data. On the one hand, with the individual sets of customer requests forming the baseline for the simulation, their definition as a significant input on the simulation's output. If these future scenarios do not represent the real upcoming market and business evolvement, the simulation result may differ.

Furthermore, if the evaluation of the need for change and the resulting change efforts differ significantly from the real evolving future, the simulation's results can also be misleading. In order to cope with that issue, one possible solution is to focus only on the most relevant change drivers, in order to reduce the necessary effort as well as the impact of non-first-priority drivers, as these are more susceptible to misleading future scenarios. An approach related to the pareto-method should be carried out, implementing only the most relevant $20 \%$ of change drivers, which tend to represent around $80 \%$ of the change impact.

On the other hand, even if the scenario analysis for future changes does not comply with the real evolving future, the DfFR-alternative is undoubtedly more performant than the original status quo product architecture, a significant performance improvement can be achieved anyway.

\section{CONCLUSION AND OUTLOOK}

As a conclusion, it can be said that the presented simulation method proposes a well-suited visual and mathematical approach to compare different product architectures. The main advantage is the adaptability to the current use case scenario as shown during this contribution. It also can be used as a tool to evaluate the performance of product architectures adapted to future scenarios, enabling an early-stage performance assessment without the need of major time- and capacity investments. The results show clear and obvious, that the DfV-alternative shows a significant advantage over the 
original status quo alternative, which is according to the expectance as the internal variety is majorly reduced. Furthermore, the DfFR-alternative shows a further performance improvement due to the increase in the external variety towards the customer and the future-robust standardisation. Nevertheless, these efforts still result in an increase of the manufacturing costs, slightly reducing the product architecture's performance. On the other hand, the overall performance is still high. This results in the decision, that in this particular case over-dimensioning does not impact the overall performance as negatively as predicted. As a future research area, the applied configuration system as a core simulation element can be expanded in order to enable a Design-to-Cost as well as Design-toUse approach for the already existing, implemented product architecture. By collecting the configuration data over a certain period of time, keeping track of the configuration frequency of individual modules enables an assessment, which modules get configured most. As these modules therefore will be used most, optimizing their internal set-up in terms of manufacturing efficiency as well as component performance and cost is crucial. Furthermore, when comparing the configured product variant with the real manufactured and specifically to each customer adapted product variant, a further optimization of the product architecture can be achieved.

\section{REFERENCES}

Bauer, W. (2016), Planung und Entwicklung änderungsrobuster Plattformarchitekturen, Ph.D. thesis, Technische Universität München.

Cicconi, P.; Raffaeli, R.; Marchionne, M.; Germani, M. (2018): A model-based simulation approach to support the product configuration and optimization of gas turbine ducts, Computer-Aided Design \& Applications, Vol. 15, pp. 807-818, https://doi.org/10.1080/16864360.2018.1462564.

Fink, A. and Siebe, A. (2016), Szenario Management: Von strategischem Vorausdenken zu zukunftsrobusten Entscheidungen, Campus, Frankfurt a. M., Germany.

Greve, E. and Krause, D. (2018), “An Assessment of Methods to support the design of future robust modular product architectures”, Proceedings of the Design Society: DESIGN Conference, Dubrovnik, Croatia, pp. 335-346. https://doi.org/10.21278/idc.2018.0249

Greve, E.; Fuchs, C.; Hamraz, B.; Windheim, M.; Krause, D. (2021), "Design for future Variety to enable longterm Benefits of Modular Product Families”, 23rd International Conference on Engineering Design (ICED21), Gothenburg, Sweden, August 16-20.

Hackl, J., Krause, D., Otto, K., Windheim, M., Moon, S.K., Bursac, N. and Lachmayer, R. (2020), "Impact of Modularity Decisions on a Firm's Economic Objectives ”, Journal of Mechanical Design, Vol. 142 No. 4. https://doi.org/10.1115/1.4044914

James, T. J. Y.; Otto, K.; Wood, K. L. (2016): Design Concept Screening in Compex System Using Component Constraints, Proceedings of the International Design Engineering Technical Conferences \& Computers and Information in Engineering Conference (IDETC 2016), Charlotte, North Carolina.

Krause, D. and Gebhardt, N. (2018): Methodische Entwicklung modularer Produktfamilien, Springer, Berlin, https://doi.org/10.1007/978-3-662-53040-5.

Kipp, T. and Krause, D. (2008), "Design for Variety: Efficient Support for Design Engineers ", Proceedings of the Design Society: DESIGN Conference, Dubrovnik, Croatia, pp. 425-432.

Kovalenko, I.; Tilbury, D.; Barton, K. (2019): The model-based product agent: A control oriented architecture for intelligent products in multi-agent manufacturing systems, Control Engineering Practice, Vol. 86, pp. 105-117, https://doi.org/10.1016/j.conengprac.2019.03.009.

Salvador, F. (2007), “Towards a product system modularity construct: Literature review and reconceptualization”, IEEE Transactions on Engineering Management, Vol. 54 No. 2. https://dx.doi/org/10.1109/TEM.2007.893996

Seiler, F. M. and Krause, D. (2020a): A Multi-Dimensional Configuration Algorithm for Modular Product Architectures, Proceedings of the Design Society: DESIGN Conference, Vol. 1, Dubrovnik, Croatia, pp. 2405-2414. https://doi.org/10.1017/dsd.2020.283

Seiler, F. M.; Kuhl, J.; Krause, D. (2020b): A Simulation-Based Decision Support Method For Modular Product Architecture Alternatives, 22nd International Dependency and Structure Modeling Conference (DSM 2020), Cambridge, MA, USA, https://doi.org/10.35199/dsm2020.9.

Stjepandic, J.; Wognum, N.; Verhagen, W. (2015): Concurrent Engineering in the 21st Century: Foundations, Developments and Challenges, Springer International, Cham, https://doi.org/10.1007/978-3-319-13776-6.

Zennaro, I.; Finco, S.; Battini, D.; Persona, A. (2019): Big size highly customised product manufacturing systems: a literature review and future research agenda, International Journal of Production Research, Vol. 57 (15-16), pp. 5362-5385, https://doi.org/10.1080/00207543.2019.1582819. 